\title{
Investigating the Use of Speech Act of Suggestions of Turkish ELT Students
}

\author{
Müfit Şenel \\ Ondokuz Mayıs University, Turkey.e-mail:mufitsnl@gmail.com
}

\begin{tabular}{|c|c|}
\hline ARTICLE INFO & ABSTRACT \\
\hline $\begin{array}{l}\text { Keywords: } \\
\text { speech act, suggestion, } \\
\text { ELT, written discourse } \\
\text { completion task }\end{array}$ & $\begin{array}{l}\text { This paper outlines an attempt to research the speech act of suggestion of ELT } \\
\text { students majoring at a state university in Turkey. A written discourse } \\
\text { completion task (WDCT) and a semi-structured interview were used to collect } \\
\text { data. The WDCT was developed by the researcher, and the responses of the } \\
\text { participants were analyzed based on Martinez-Flor's speech act of suggestion } \\
\text { criteria. Regarding the participants' answers, it can be said that most of the } \\
\text { participants used more conventionalized forms rather than the other forms. } \\
\text { The data revealed that 'direct strategies' were the least used ones, but 'hints' } \\
\text { were never used. Moreover, gender and high school differences did not play a } \\
\text { prominent role in the production of suggestion strategies. It is believed that } \\
\text { the findings of this study will enlighten our knowledge to understand some } \\
\text { Turkish EFL/ELT university students'production and perception of the speech } \\
\text { act of suggestion because most of the studies in the Turkish context were } \\
\text { about either refusal or complaining strategies; therefore, this study is believed } \\
\text { to fill in this gap in this area. Correspondingly, this present study will also pave } \\
\text { the ground to reconsider some critical points regarding the gap in the } \\
\text { literature. }\end{array}$ \\
\hline
\end{tabular}

How to cite:

Şenel, M. (2021). Investigating the Use of Speech Act of Suggestions of Turkish ELT Students. Indonesian Journal of English Language Teaching and Applied Linguistics, 6(1), 27-43

\section{Introduction}

It is inevitable for any society to encounter different types of situations such as requesting, apologizing, turn-taking, addressing, requesting, ordering, giving suggestions, etc. "What people should do" or "how people should speak" and "should behave", maybe the most frequently used speech act types in society. Therefore, giving suggestions is highly prominent in people's daily lives since they regularly use and receive suggestions in their daily interactions. For example, parents suggest their children study hard, have their breakfast, lunch, and dinner, sleep early; doctors suggest their patients use a medicine, take care of their health; teachers suggest their students study hard and do their homework or we may suggest our friends travel, watch a movie, read a book, etc. It is essential to use speech acts in 
foreign/second language contexts because speech acts are the crucial elements of effective communication and they reflect the fundamental values, social and cultural norms of the target language community. Additionally, classrooms are among the leading settings where students ask for teachers' help and teachers advise their students. Non-native speakers may not be acquainted with the different effects of direct, indirect, or conventionalised forms of suggestions; therefore, non-native speakers should be cautious and informed about the intricacies of the suggestion speech act.

When the relevant literature was scrutinized, no similar studies were found for Turkish ELT university students' speech act of suggestions. Therefore, regarding the scary of studies focusing on Turkish ELT students' speech act of suggestions, this present study attempted to probe the non-native speakers of ELT students' speech act of suggestions at a state university in terms of some variables.

\subsection{Written Discourse Completion Task and Speech Act of Suggestion}

One of the means to glean, according to Cohen (1996), well-prepared and well-designed Discourse Completion Task (DCT) can help people activate their pragmatic knowledge (Martinez-Flor, 2005). In the literature, generally, six types of DCTs have been classified: Written Discourse Completion Task (WDCT), Multiple-choice Discourse Completion Task $(\mathrm{MDCT})$, Oral Discourse Completion Task (ODCT), Discourse Role-Play Task (DRPT), Discourse Self-Assessment Task (DSAT), Role-Play Self-Assessment (RPSA) (Blum-Kulka, 1982; Brown, 2001).

WDCT was defined by Brown (2001) as "any pragmatics instrument that requires the students to read a written description of a situation including factors such as setting, roles of participants, and degree of imposition and asks them to write what they would say in that situation" (p.301). Another definition for discourse completion tasks was given by Kasper \& Dahl (1991, p.221) as "written questionnaires including some brief situational descriptions followed by a short dialogue with an empty slot for the speech act under study.

In general, speech acts are classified under three basic categories: locutionary acts, illocutionary acts, and perlocutionary acts (Grundy, 2008). According to Schmidt \& Richards (1980), all the acts we do while speaking are parts of speech acts. The main contribution of speech act theory to the language teaching field is the explanation of communicative competence.

\section{Literature Review}

Generally speaking, speech act studies in the literature have been tabulated under two categories: First group of studies conducted by Al-kahtani (2005); Cheng (2009); Karimnia \& Afghari (2010) and Sharifian, (2005) dealt with the native speakers while Ahmadian \& Vahid Dastjerdi (2010); Al-Eryani (2007); Bryant Smith (2009); Parvaresh \& Eslami Rasekh (2009); Wannaruk (2008) and Wolfson (1981) studied with non-native speakers in comparison to their preferences with native speakers. A great deal of studies has been carried out regarding different types of speech acts by means of WDCT. For example, requesting (Jalilifar, 2009; Taguchi, 2006), apologizing (Eslami-Rasekh \& Mardani, 2010; Harris et al., 2006), complimenting (Sharifian, 2008; Wolfson, 1981), thanking, requesting, promising (Marquez Reiter, 2000) and refusing (Allami \& Naeimi, 2010; Tanck, 2002). Additional studies were conducted by Pishghadam \& Sharafadini (2011a), Allami (2006); Allami \& Naeimi (2010); 
Bryant Smith (2009); Nguyen (2007); Sumhung Li (2010); Wannaruk (2008); Alzeebaree \& Yavuz (2017); Amelia Maldin (2019); Lenchuk \& Ahmed (2019); Nugroho et al., (2018); Sabzalipour et al., (2017) and Shofwan \& Mujiyanto, (2018).

A comparative study of gender differences in refusal strategies from English majors surveyed by Wang (2019) enlightened the fact that the majority of the female participants used indirect refusal politeness strategies to avoid the face-threatening, while most of the male students seemed to use direct refusals. Christianto (2020) declared three types of speech acts found in the interactions between the teachers and students in EFL classes. To criticize the university teachers' talk with their students regarding the realization of speech acts, the researchers concluded that teachers generally preferred direct strategies and the minimal use of modifiers (Salam El-Dakhs et al., 2019). Similarly, according to a speech act analysis of teacher talk in EFL classes, it was mentioned that teachers mostly use directive speech acts because they adopted the principles of CLT (Basra \& Thoyyibah, 2017).

Farnia et al.'s (2014) research unearthed that Iranian native speakers of Farsi used more directive strategies. Furthermore, a comparative study on speech acts by native speakers and Turkish learners of English indicated that native English speakers' and Turkish learners' use of complaints showed a statistically significant difference concerning the linguistic components and the pragmatic choices (Önalan \& Çakır, 2018). Aminifard et al. (2014) sought to shed light on how Iranian EFL learners employed the suggestion speech act. Results highlighted that although the participants had different language proficiency levels, any significant differences in the production of suggestion speech acts were not found. However, in terms of gender, participants' performances showed statistical significance. Shofwan \& Mujiyanto (2018) unearthed that EFL learners used conventionalized forms more often than other strategies. Yelfiza (2013) reported that the types of speech acts uttered by the lectures were praising, questioning, asserting, etc., and the selection of those types was all influenced by the culture of the lecturers.

Sharqawi \& Anthony (2019) explained that although male and female participants employed similar amounts of explicit disagreement strategies, female participants significantly used more indirect disagreement strategies than male participants. Correspondingly, Sharkqawi \& Anthony again (2020) analyzed the role of gender on the speech act of suggestion, and they announced that females had more indirect suggestions than males. Depending upon a corpus-based study on the performance of the suggestion speech act by Chinese EFL learners, Gu (2014) reported that they used significantly more conventionalized indirect suggestion strategies than native English speakers. Shahreza (2014) attempted to crystallize the speech act of suggestion of Iranian EFL learners, and he revealed that male students tended to be significantly more direct than their female students. In line with these studies, Yıldız (2020) dealt with the use of suggestion strategies among Turkish EFL students and opined that most of the participants had used "should" and "possibility" as suggestion strategies. From the perspective of the Turkish EFL context, Güngörmezler (2016) compared and contrasted the refusal speech act of suggestions of Turkish and American speakers, and the results put forward that providing excuse/reason/explanation was the most preferred strategy for both groups. A gender-focused research to investigate the refusal strategies of Turkish pre-service teachers of English conducted by Tuncer \& Burhan (2019) revealed that excuse/reason/explanation was among the most used refusal strategies. 


\section{Purpose of the study}

This study aimed to investigate the realization of the speech act of suggestion of some Turkish university students at ELT department regarding the dearth of studies in the Turkish context. Answers were sought for the following research questions: Is there any significant difference between:

1. males and females?

2. participants graduated from different high schools?

3. the grades of the students?

4. the ages of participants?

The reasons for selecting those variables can be explained as mentioned here. Since females and males have different social statuses in society and the type of language is generally different, gender was selected as the first variable. Secondly, graduated high schools were another variable because high schools in Turkey follow somewhat different language programs. The third variable was the grades of the participants because they follow different courses at the faculty and the final one was the ages of the participants because the young generation generally uses different language than elders.

\section{Research Methodology}

\subsection{Participants}

For the concern of this study, 158 university students majoring in the ELT department of a state university in Turkey were invited. They could actively speak and write in English at B1 level regarding their university entrance grades. Their demographic distribution was given below (Table 1).

Table 1: Demographic distribution of participants

\begin{tabular}{|c|c|c|c|}
\hline & & $f$ & $\%$ \\
\hline \multirow[t]{2}{*}{ Gender } & Female & 106 & 67.1 \\
\hline & Male & 52 & 32.9 \\
\hline \multirow[t]{2}{*}{ Grade } & 3 & 62 & 39.2 \\
\hline & 4 & 96 & 60.8 \\
\hline High & Anatolian High School & 89 & 56.3 \\
\hline \multirow[t]{2}{*}{ School } & $\begin{array}{l}\text { Anatolian İmam Hatip High School } \\
\text { Anatolian Teacher's High School }\end{array}$ & 34 & 21.5 \\
\hline & & 35 & 22.2 \\
\hline \multirow[t]{2}{*}{ Age } & $19-21$ & 78 & $49 \cdot 4$ \\
\hline & $22-24$ & 80 & 50.6 \\
\hline Total & & 158 & 100 \\
\hline
\end{tabular}

\subsection{Data Collection}

The data were collected during the 2019-2020 winter semester at the last 20-25 minutes of participants' class hours with the permission of their teachers by means of two instruments: A written discourse completion task (WDCT) including 10 open-ended questions and a semistructured interview with four questions. 


\subsubsection{Written Discourse Completion Task}

Written Discourse Completion Task (WDCT), adapted from Pishghadam and Sharafadini's (2011a) study, was used to obtain data about participants' use of suggestions in different situations. It was modified, rewritten and designed based on the guidelines provided by Banerjee \& Carrell (1988), Martinez-Flor (2006), Martinez-Flor (2005), Martinez-Flor \& Alcon Soler (2004), Martinez-Flor \& Fukuya (2005). It was piloted to 17 ELT learners at a level commensurate to that of the participants by the researchers to spot any possible ambiguous item. No possible ambiguous items were found. Additionally, 10 ELT teachers at the same department were asked to substantiate the content validity of the WDCT. Its validity was clarified as.87.

\subsubsection{Semi-structured interview}

As a second data collection instrument, the following questions were delivered to the participants, and they were asked to write their responses within an allocated time (10-15 minutes).

1. What did you pay attention to when you wanted to suggest somebody?

2. Did your response change if the person in the situation were younger or older than you?

3. Were your responses different if the person in the situation was a close friend or a stranger?

4. Did the gender of the interlocutor influence your response?

\subsection{Procedure}

Quantitative and qualitative research methods were used. The WDCT with ten open-ended questions was distributed among participants, and they were asked to write their suggestions as if they were in those situations. Participants were also informed that all their responses would only be used for that research and would not be shared and published anywhere. Necessary explanations were given in the native language of the participants to eliminate misunderstanding (Martinez-Flor \& Alcon-Soler, 2004). Data collected by WDCT were analyzed according to the taxonomy of the suggestion strategies proposed by Martinez-Flor (2005). Possible suggestions had been classified under three basic degrees:

1. direct forms (performative verb, noun of suggestions, imperative, and negative imperative).

2. conventionalized forms (interrogative forms (specific formulae), possibilityprobability, should, need, and conditional).

3. indirect forms (impersonal verbs and hints).

After collecting the data, responses of the participants were coded from 1 to 11 according to the types of the words, phrases or expressions that they had used: (performative verb:1; noun of suggestions: 2 ; imperative: 3 ; negative imperative: $4 ;$ interrogative form: 5 ; possibilityprobability: 6; should: 7; need: 8; conditional: 9; impersonal:10 and hints:11) Later, responses of the participants were analyzed and assessed quantitatively by SPSS 23 statistical package program. The collected data were analyzed by using descriptive statistics. The findings were presented separately for each sub-level of the variables to determine whether there is a relationship and dependency between the variables and participants' utilization of different suggestion forms. 
After the implementation of the WDCT, the semi-structured interview was conducted to obtain and clarify the responses of the volunteer 27 participants' information about the speech act of suggestions.

\section{Findings}

All WDCT data were analyzed against Martinez-Flor's (2005) coding scheme of speech act of suggestion. Table 2 represents the results of the suggestion strategies elicited from all participants.

Table 2: General classification of the participants' responses

\begin{tabular}{|c|c|c|c|c|c|c|c|c|c|c|}
\hline & \multicolumn{2}{|c|}{ Q1 } & \multicolumn{2}{|c|}{ Q2 } & \multicolumn{2}{|c|}{ Q3 } & \multicolumn{2}{|c|}{$\mathrm{O}_{4}$} & \multicolumn{2}{|c|}{$\mathrm{Q}_{5}$} \\
\hline & f & $\%$ & $f$ & $\%$ & $f$ & $\%$ & $f$ & $\%$ & f & $\%$ \\
\hline Performative verb & 40 & $25 \cdot 3$ & 26 & 16.5 & 34 & 21.5 & 19 & 12.0 & 11 & 7.0 \\
\hline $\begin{array}{l}\text { Nouns of } \\
\text { suggestions }\end{array}$ & 15 & $9 \cdot 5$ & - & - & - & - & 1 & 6 & 42 & 26.6 \\
\hline Imperative & 1 & 6 & 34 & 21.5 & 1 & 6 & - & - & - & - \\
\hline Negative imperative & - & - & - & - & - & - & 25 & 15.8 & - & - \\
\hline Interrogative form & 24 & 15.2 & 18 & 11.4 & 28 & 17.7 & 10 & 8.3 & 31 & 19.6 \\
\hline $\begin{array}{l}\text { Possibility- } \\
\text { probability }\end{array}$ & 19 & 12.0 & 23 & 14.6 & 23 & 14.6 & 31 & 19.6 & 3 & 1.9 \\
\hline Should & 1 & 6 & 8 & 5.1 & 22 & 13.9 & 25 & 15.8 & 38 & 24.1 \\
\hline Need & 23 & 14.6 & 4 & 2.5 & 14 & 8.9 & 7 & $4 \cdot 4$ & 4 & 2.5 \\
\hline Conditional & 14 & 8.9 & 15 & $9 \cdot 5$ & 22 & 13.9 & 27 & 17.1 & 14 & 8.9 \\
\hline Impersonal & 21 & $13 \cdot 3$ & 30 & 19.0 & 14 & 8.9 & 13 & 8.2 & 13 & 8.2 \\
\hline Hints & - & - & - & - & - & - & - & - & - & - \\
\hline Missing answers* & - & - & - & - & - & - & - & - & 2 & 1.3 \\
\hline \multirow[t]{3}{*}{ Total } & 158 & 100 & 158 & 100 & 158 & 100 & 158 & 100 & 158 & 100 \\
\hline & \multicolumn{2}{|c|}{ Q6 } & \multicolumn{2}{|c|}{ Q7 } & \multicolumn{2}{|c|}{ Q8 } & \multicolumn{2}{|c|}{ Q9 } & \multicolumn{2}{|c|}{ Q10 } \\
\hline & $f$ & $\%$ & $f$ & $\%$ & $f$ & $\%$ & $f$ & $\%$ & $f$ & $\%$ \\
\hline Performative verb & 8 & 5.1 & 12 & 7.6 & 23 & 14.6 & 17 & 10.8 & 6 & 3.8 \\
\hline $\begin{array}{l}\text { Nouns of } \\
\text { suggestions }\end{array}$ & 10 & 6.3 & 19 & 12.0 & 3 & 1.9 & 1 & 6 & 14 & 8.9 \\
\hline Imperative & 37 & 23.4 & 14 & 8.9 & 2 & 1.3 & 1 & 6 & 1 & 6 \\
\hline Negative imperative & - & - & 27 & 17.1 & 24 & 15.2 & 11 & 7.0 & - & - \\
\hline Interrogative form & 21 & $13 \cdot 3$ & 7 & $4 \cdot 4$ & 27 & 17.1 & 37 & 23.4 & 13 & 8.2 \\
\hline $\begin{array}{l}\text { Possibility- } \\
\text { probability }\end{array}$ & 9 & $5 \cdot 7$ & 27 & 17.1 & 9 & $5 \cdot 7$ & 5 & 3.2 & 2 & 1.3 \\
\hline Should & 19 & 12.0 & 17 & 10.8 & 20 & 12.7 & 31 & 19.6 & 26 & 16.5 \\
\hline Need & 7 & $4 \cdot 4$ & 2 & 1.3 & 21 & $13 \cdot 3$ & 5 & 3.2 & 10 & 6.3 \\
\hline Conditional & 14 & 8.9 & 22 & 13.9 & 13 & 8.2 & 23 & 14.6 & 41 & 25.9 \\
\hline Impersonal & 23 & 14.6 & 6 & 3.8 & 16 & 10.1 & 27 & 17.1 & 43 & 27.2 \\
\hline Hints & - & - & - & - & - & - & - & - & - & - \\
\hline Missing answers* & 10 & 6.3 & 5 & 3.8 & - & - & - & - & - & - \\
\hline Total & $15^{8}$ & 100 & 158 & 100 & 158 & 100 & 158 & 100 & 158 & 100 \\
\hline
\end{tabular}

The findings show that majority of the participants use direct strategies for $\mathrm{O}_{1}, \mathrm{O}_{2}, \mathrm{O}_{3}, \mathrm{O}_{5}$, Q6 and Q7. On the other hand, 43 (27.2\%) participants use indirect (impersonal) strategies for Q10. Notably, it is also interesting that none of the participants preferred using "hints" for 
their answers because hints may be deliberately ambiguous to give the hearer 'a way out' and are fairly common in classroom language for Turkish students. Additionally, the least preferred strategy was "negative imperative" among the participants.

\subsection{Findings for the first research question}

For the first research question, it can be stated that there is a significant difference between female and male participants' preferences in terms of their use of suggestions (Table 3 and 4).

Tablez: Preferences of the participants in terms of their gender

\begin{tabular}{|c|c|c|c|c|c|c|c|c|c|c|c|}
\hline $\begin{array}{l}\text { Suggestion } \\
\text { types }\end{array}$ & Strategy & $\begin{array}{l}\mathrm{Q}_{1} \\
(f)\end{array}$ & $\begin{array}{l}\mathrm{Q}_{2} \\
(f)\end{array}$ & $\begin{array}{l}\mathrm{O}_{3} \\
(f)\end{array}$ & $\begin{array}{l}\mathrm{O}_{4} \\
(f)\end{array}$ & $\begin{array}{l}\mathrm{Q}_{5} \\
(f)\end{array}$ & $\begin{array}{l}\text { Q6 } \\
(f)\end{array}$ & $\begin{array}{l}\text { Q7 } \\
(f)\end{array}$ & $\begin{array}{l}\text { Q8 } \\
(f)\end{array}$ & $\begin{array}{l}\mathrm{Og}_{9} \\
(f)\end{array}$ & $\begin{array}{l}\mathrm{Q}_{1} \\
\mathrm{O} \\
(f)\end{array}$ \\
\hline \multirow{4}{*}{ Direct } & Performative Verb & 10 & 8 & 13 & 4 & 3 & 3 & 2 & 6 & 5 & 2 \\
\hline & $\begin{array}{l}\text { Noun of } \\
\text { Suggestions }\end{array}$ & 3 & - & - & 1 & 17 & 3 & 6 & 1 & - & 4 \\
\hline & Imperative & - & 11 & - & - & - & 11 & 6 & - & - & - \\
\hline & $\begin{array}{l}\text { Negative } \\
\text { Imperative }\end{array}$ & - & - & - & 9 & - & - & 10 & 8 & 5 & - \\
\hline \multirow{5}{*}{$\begin{array}{l}\text { Conventionaliz } \\
\text { ed forms }\end{array}$} & Interrogative Form & 5 & 4 & 9 & 2 & 6 & 10 & 1 & 9 & 12 & 3 \\
\hline & $\begin{array}{l}\text { Possibility/Probabili } \\
\text { ty }\end{array}$ & 4 & 11 & 13 & 12 & - & 2 & 13 & 2 & 2 & - \\
\hline & Should & - & 2 & 7 & 11 & 16 & 7 & 3 & 9 & 6 & 12 \\
\hline & Need & 13 & 2 & 4 & 4 & - & 2 & 1 & 7 & 1 & 2 \\
\hline & Conditional & 8 & 6 & 4 & 5 & 4 & 5 & 4 & 4 & 11 & 14 \\
\hline \multirow[t]{4}{*}{ Indirect } & Impersonal & 9 & 8 & 2 & 4 & 5 & 7 & 3 & 6 & 10 & 14 \\
\hline & Hints & - & - & - & - & - & - & - & - & - & - \\
\hline & Missing answers* & - & - & - & - & 1 & 2 & 3 & - & - & 1 \\
\hline & Total & 52 & 52 & 52 & 52 & 51 & 50 & 49 & 52 & 52 & 51 \\
\hline
\end{tabular}

* Number of the participants who had not written any answers

Table4: Preferences of the participants in terms of their gender

\begin{tabular}{|c|c|c|c|c|c|c|c|c|c|c|c|}
\hline $\begin{array}{l}\text { Suggestion } \\
\text { types }\end{array}$ & Strategy & $\begin{array}{l}\mathrm{Q}_{1} \\
(f)\end{array}$ & $\begin{array}{l}\mathrm{Q}_{2} \\
(f)\end{array}$ & $\begin{array}{l}\mathrm{Q}_{3} \\
(f)\end{array}$ & $\begin{array}{l}\mathrm{O}_{4} \\
(f)\end{array}$ & $\begin{array}{l}\mathrm{Q}_{5} \\
(f)\end{array}$ & $\begin{array}{l}\text { Q6 } \\
(f)\end{array}$ & $\begin{array}{l}\text { Q7 } \\
(f)\end{array}$ & $\begin{array}{l}\text { Q8 } \\
(f)\end{array}$ & $\begin{array}{l}\text { Q9 } \\
(f)\end{array}$ & $\begin{array}{l}\mathrm{Q}_{1} \\
\mathrm{O} \\
(f)\end{array}$ \\
\hline \multirow{4}{*}{ Direct } & Performative Verb & 30 & 18 & 21 & 15 & 8 & 5 & 10 & 17 & 12 & 4 \\
\hline & $\begin{array}{l}\text { Noun of } \\
\text { Suggestions }\end{array}$ & 12 & - & - & - & 25 & 7 & 13 & 2 & 1 & 10 \\
\hline & Imperative & 1 & 23 & 1 & - & - & 26 & 8 & 2 & 1 & 1 \\
\hline & $\begin{array}{l}\text { Negative } \\
\text { Imperative }\end{array}$ & - & - & - & 16 & - & - & 17 & 16 & 6 & - \\
\hline \multirow{5}{*}{$\begin{array}{l}\text { Conventionaliz } \\
\text { ed forms }\end{array}$} & Interrogative Form & 19 & 14 & 19 & 8 & 25 & 11 & 6 & 18 & 25 & 10 \\
\hline & $\begin{array}{l}\text { Possibility/Probabili } \\
\text { ty }\end{array}$ & 15 & 12 & 10 & 19 & 3 & 7 & 14 & 7 & 3 & 2 \\
\hline & Should & 1 & 6 & 15 & 14 & 22 & 12 & 14 & 11 & 25 & 14 \\
\hline & Need & 10 & 2 & 10 & 3 & 4 & 5 & 1 & 14 & 4 & 8 \\
\hline & Conditional & 6 & 9 & 18 & 22 & 10 & 9 & 18 & 9 & 12 & 27 \\
\hline \multirow[t]{5}{*}{ Indirect } & Impersonal & 12 & 22 & 12 & 9 & 8 & 16 & 3 & 10 & 17 & 29 \\
\hline & Hints & - & - & - & - & - & - & - & - & - & - \\
\hline & Missing answers* & - & - & - & - & 1 & 8 & 2 & - & - & 1 \\
\hline & Total & 10 & 10 & 10 & 10 & 105 & 98 & 10 & 10 & 10 & 105 \\
\hline & & 6 & 6 & 6 & 6 & & & 4 & 6 & 6 & \\
\hline
\end{tabular}

* Number of the participants who had not written any answers 
When Tables 3 and 4 are examined, it is seen that both females and males preferred the use of conventionalized forms of suggestion ( $53.4 \% \mathrm{~F}, 50 \% \mathrm{M}$ ). Additionally, for females, the frequency of direct strategies is $33.5 \%$, and for males, it is $33.7 \%$. To put it briefly, findings display that there is no specific distinction between female and male participants' preferences in their types of strategies.

\subsection{Findings for the second research question}

For research question 2, tables 5, 6 and 7 provide confirmatory evidence explained below.

Table 5: Preferences of the participants in terms of their high schools

\begin{tabular}{|c|c|c|c|c|c|c|c|c|c|c|c|}
\hline Suggestion types & Strategy & $\begin{array}{l}\mathrm{Q}_{1} \\
(f)\end{array}$ & $\begin{array}{l}\mathrm{Q}_{2} \\
(f)\end{array}$ & $\begin{array}{l}\mathrm{O}_{3} \\
(f)\end{array}$ & $\begin{array}{l}\mathrm{O}_{4} \\
(f)\end{array}$ & $\begin{array}{l}\mathrm{Q}_{5} \\
(f)\end{array}$ & $\begin{array}{l}\text { Q6 } \\
(f)\end{array}$ & $\begin{array}{l}\text { Q7 } \\
(f)\end{array}$ & $\begin{array}{l}\text { Q8 } \\
(f)\end{array}$ & $\begin{array}{l}\text { Qg } \\
(f)\end{array}$ & $\begin{array}{l}\text { Q10 } \\
(f)\end{array}$ \\
\hline \multirow{4}{*}{ Direct } & Performative Verb & 23 & 13 & 23 & 10 & 6 & 5 & 7 & 11 & 10 & 5 \\
\hline & Noun of Suggestions & 8 & - & - & 1 & 24 & 6 & 12 & 3 & 1 & 8 \\
\hline & Imperative & 1 & 22 & 1 & - & - & 16 & 8 & 2 & 1 & 1 \\
\hline & Negative Imperative & - & - & - & 16 & - & - & 11 & 15 & 6 & - \\
\hline \multirow{5}{*}{$\begin{array}{l}\text { Conventionalized } \\
\text { forms }\end{array}$} & Interrogative Form & 11 & 10 & 15 & 6 & 12 & 16 & 3 & 13 & 17 & 7 \\
\hline & Possibility/Probability & 12 & 12 & 13 & 17 & 2 & 4 & 18 & 4 & 5 & 1 \\
\hline & Should & - & 5 & 9 & 16 & 21 & 10 & 6 & 10 & 18 & 11 \\
\hline & Need & 11 & 3 & 7 & 4 & 2 & 5 & 2 & 13 & 3 & 5 \\
\hline & Conditional & 6 & 6 & 14 & 12 & 10 & 10 & 16 & 9 & 13 & 22 \\
\hline \multirow[t]{4}{*}{ Indirect } & Impersonal & 17 & 18 & 7 & 7 & 11 & 11 & 5 & 9 & 15 & 28 \\
\hline & Hints & - & - & - & - & - & - & - & - & - & - \\
\hline & Missing answers* & - & - & - & - & 1 & 6 & 1 & - & - & 1 \\
\hline & Total & 89 & 89 & 89 & 89 & 88 & 83 & 89 & 89 & 89 & 88 \\
\hline
\end{tabular}

*Number of the participants who had not written anything

Table 6: Preferences of the participants in terms of their high schools

\begin{tabular}{|c|c|c|c|c|c|c|c|c|c|c|c|c|}
\hline & Suggestion types & Strategy & $\begin{array}{l}\mathrm{Q}_{1} \\
(f)\end{array}$ & $\begin{array}{l}\mathrm{Q}_{2} \\
(f)\end{array}$ & $\begin{array}{l}\mathrm{Q}_{3} \\
(f)\end{array}$ & $\begin{array}{l}\mathrm{O}_{4} \\
(f)\end{array}$ & $\begin{array}{l}\mathrm{Q}_{5} \\
(f)\end{array}$ & $\begin{array}{l}\text { Q6 } \\
(f)\end{array}$ & $\begin{array}{l}\text { Q7 } \\
(f)\end{array}$ & $\begin{array}{l}\text { Q8 } \\
(f)\end{array}$ & $\begin{array}{l}\text { Q9 } \\
(f)\end{array}$ & $\begin{array}{l}\mathrm{Q}_{10} \\
(f)\end{array}$ \\
\hline \multirow{13}{*}{ 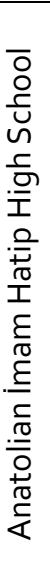 } & \multirow{4}{*}{ Direct } & Performative Verb & 7 & 5 & 4 & 5 & - & 1 & 2 & 5 & 5 & 1 \\
\hline & & Noun of Suggestions & 4 & - & - & - & 13 & 1 & 2 & - & - & 4 \\
\hline & & Imperative & - & 6 & - & - & - & 11 & 4 & - & - & - \\
\hline & & Negative Imperative & - & - & - & 6 & - & - & 8 & 4 & 2 & - \\
\hline & \multirow{5}{*}{$\begin{array}{l}\text { Conventionalized } \\
\text { forms }\end{array}$} & Interrogative Form & 6 & 4 & 11 & 2 & 10 & 5 & 2 & 6 & 10 & 2 \\
\hline & & Possibility/Probability & 5 & 6 & 6 & 9 & - & 1 & 5 & 3 & - & 1 \\
\hline & & Should & - & 2 & 6 & 4 & 7 & 4 & 6 & 7 & 8 & 8 \\
\hline & & Need & 6 & - & 1 & - & 2 & 1 & - & 4 & 1 & 3 \\
\hline & & Conditional & 5 & 4 & 3 & 6 & 2 & 2 & 2 & 2 & 3 & 6 \\
\hline & \multirow[t]{4}{*}{ Indirect } & Impersonal & 1 & 7 & 3 & 2 & - & 6 & 1 & 3 & 5 & 9 \\
\hline & & Hints & - & - & - & - & - & - & - & - & - & - \\
\hline & & Missing answers* & - & - & - & - & - & 2 & 2 & - & - & - \\
\hline & & Total & 34 & 34 & 34 & 34 & 34 & 32 & 32 & 34 & 34 & 34 \\
\hline
\end{tabular}

*Number of the participants who had not written anything 
Table 7: Preferences of the participants in terms of their high schools

\begin{tabular}{|c|c|c|c|c|c|c|c|c|c|c|c|}
\hline $\begin{array}{l}\text { Suggestion } \\
\text { types }\end{array}$ & Strategy & $\begin{array}{l}\mathrm{Q}_{1} \\
(f)\end{array}$ & $\begin{array}{l}\mathrm{Q}_{2} \\
(f)\end{array}$ & $\begin{array}{l}\mathrm{O}_{3} \\
(f)\end{array}$ & $\begin{array}{l}\mathrm{O}_{4} \\
(f)\end{array}$ & $\begin{array}{l}\mathrm{Q}_{5} \\
(f)\end{array}$ & $\begin{array}{l}\text { Q6 } \\
(f)\end{array}$ & $\begin{array}{l}\text { Q7 } \\
(f)\end{array}$ & $\begin{array}{l}\text { Q8 } \\
(f)\end{array}$ & $\begin{array}{l}\text { Q9 } \\
(f)\end{array}$ & $\begin{array}{l}\text { Q10 } \\
(f)\end{array}$ \\
\hline \multirow{4}{*}{ Direct } & Performative Verb & 10 & 8 & 7 & 4 & 5 & 2 & 3 & 7 & 2 & - \\
\hline & Noun of Suggestions & 3 & - & - & - & 5 & 3 & 5 & - & - & 2 \\
\hline & Imperative & - & 6 & - & - & - & 10 & 2 & - & - & - \\
\hline & Negative Imperative & - & - & - & 3 & - & - & 8 & 5 & 3 & - \\
\hline \multirow{5}{*}{$\begin{array}{l}\text { Conventionalize } \\
\text { d forms }\end{array}$} & Interrogative Form & 7 & 4 & 2 & 2 & 9 & - & 2 & 8 & 10 & 4 \\
\hline & Possibility/Probability & 2 & 5 & 4 & 5 & 1 & 4 & 4 & 2 & - & - \\
\hline & Should & 1 & 1 & 7 & 5 & 10 & 5 & 5 & 3 & 2 & 7 \\
\hline & Need & 6 & 1 & 6 & 3 & - & 1 & - & 4 & 1 & 2 \\
\hline & Conditional & 3 & 5 & 5 & 9 & 2 & 2 & 4 & 2 & 7 & 13 \\
\hline \multirow[t]{4}{*}{ Indirect } & Impersonal & 3 & 5 & 4 & 4 & 2 & 6 & - & 4 & 7 & 6 \\
\hline & Hints & - & - & - & - & - & - & - & - & - & - \\
\hline & Missing answers* & - & - & - & - & 1 & 2 & 2 & - & 3 & 1 \\
\hline & Total & 35 & 35 & 35 & 35 & 34 & 33 & 33 & 35 & 32 & 34 \\
\hline
\end{tabular}

* Number of the participants who had not written any answers

Coupled with the evidence given in tables 5, 6 and 7, the results paint a compelling view of the justification of Anatolian High School students' responses are respectively different from the responses of Anatolian İmam Hatip High School and Anatolian Teacher's High School's participants. Concerning the preferences, the participants of Anatolian Imam Hatip High School and Anatolian Teacher's High School show more similarities; that is to say, their preferences did not change although they had graduated from different high schools. Results evinced that those participants had taken similar foreign language education, reflecting the same cultural values.

\subsection{Findings for the third research question}

For research question 3 , the analysis of tables 8 and 9 lead to the following conclusions:

Table 8: Preferences of the participants in terms of their grades

\begin{tabular}{|c|c|c|c|c|c|c|c|c|c|c|c|}
\hline $\begin{array}{l}\text { Suggestion } \\
\text { types }\end{array}$ & Strategy & $\begin{array}{l}\mathrm{Q}_{1} \\
(f)\end{array}$ & $\begin{array}{l}\mathrm{O}_{2} \\
(f)\end{array}$ & $\begin{array}{l}\mathrm{Q}_{3} \\
(f)\end{array}$ & $\begin{array}{l}\mathrm{O}_{4} \\
(f)\end{array}$ & $\begin{array}{l}\mathrm{O}_{5} \\
(f)\end{array}$ & $\begin{array}{l}\text { Q6 } \\
(f)\end{array}$ & $\begin{array}{l}\text { Q7 } \\
(f)\end{array}$ & $\begin{array}{l}\text { Q8 } \\
(f)\end{array}$ & $\begin{array}{l}\text { O9 } \\
(f)\end{array}$ & $\begin{array}{l}\text { Q10 } \\
(f)\end{array}$ \\
\hline \multirow{4}{*}{ Direct } & Performative Verb & 10 & 8 & 12 & 3 & - & 1 & 1 & 6 & 6 & 3 \\
\hline & $\begin{array}{l}\text { Noun of } \\
\text { Suggestions }\end{array}$ & 6 & - & - & - & 24 & 7 & 10 & 1 & 1 & 2 \\
\hline & Imperative & 1 & 22 & 1 & - & - & 16 & 12 & 2 & 1 & 1 \\
\hline & Negative Imperative & - & - & - & 15 & - & - & 9 & 15 & 8 & - \\
\hline \multirow{5}{*}{$\begin{array}{l}\text { Conventionaliz } \\
\text { ed forms }\end{array}$} & Interrogative Form & 5 & 9 & 13 & 4 & 13 & 12 & 5 & 11 & 13 & 6 \\
\hline & $\begin{array}{l}\text { Possibility/Probabilit } \\
\text { y }\end{array}$ & 7 & 8 & 13 & 18 & - & 1 & 7 & 2 & 2 & - \\
\hline & Should & - & 1 & 5 & 9 & 16 & 3 & 6 & 10 & 12 & 8 \\
\hline & Need & 14 & 4 & 4 & 6 & - & 5 & - & 9 & 1 & 7 \\
\hline & Conditional & 8 & - & 8 & 5 & 1 & 5 & 11 & 2 & 12 & 18 \\
\hline \multirow[t]{4}{*}{ Indirect } & Impersonal & 11 & 10 & 6 & 2 & 8 & 12 & 1 & 4 & 6 & 17 \\
\hline & Hints & - & - & - & - & - & - & - & - & - & - \\
\hline & Missing answers* & - & - & - & - & - & - & - & - & - & - \\
\hline & Total & 62 & 62 & 62 & 62 & 62 & 62 & 62 & 62 & 62 & 62 \\
\hline
\end{tabular}


Table 9: Preferences of the participants in terms of their grades

\begin{tabular}{|c|c|c|c|c|c|c|c|c|c|c|c|c|}
\hline & $\begin{array}{l}\text { Suggestion } \\
\text { types }\end{array}$ & Strategy & $\begin{array}{l}\mathrm{Q}_{1} \\
(f)\end{array}$ & $\begin{array}{l}\mathrm{Q}_{2} \\
(f)\end{array}$ & $\begin{array}{l}\mathrm{Q}_{3} \\
(f)\end{array}$ & $\begin{array}{l}\mathrm{O}_{4} \\
(f)\end{array}$ & $\begin{array}{l}\mathrm{Q}_{5} \\
(f)\end{array}$ & $\begin{array}{l}\text { Q6 } \\
(f)\end{array}$ & $\begin{array}{l}\text { Q7 } \\
(f)\end{array}$ & $\begin{array}{l}\text { Q8 } \\
(f)\end{array}$ & $\begin{array}{l}\text { Q9 } \\
(f)\end{array}$ & $\begin{array}{l}\mathrm{Q}_{10} \\
(f)\end{array}$ \\
\hline \multirow{13}{*}{$\begin{array}{l}\frac{0}{0} \\
\frac{\pi}{0} \\
\frac{1}{0} \\
\stackrel{5}{+}\end{array}$} & \multirow{4}{*}{ Direct } & Performative Verb & 30 & 18 & 22 & 16 & 11 & 7 & 11 & 17 & 11 & 3 \\
\hline & & $\begin{array}{l}\text { Noun of } \\
\text { Suggestions }\end{array}$ & 9 & - & - & 1 & 18 & 3 & 9 & 2 & - & 12 \\
\hline & & Imperative & - & 12 & - & - & - & 21 & 2 & - & - & - \\
\hline & & Negative Imperative & - & - & - & 10 & - & - & 18 & 9 & 3 & - \\
\hline & \multirow{5}{*}{$\begin{array}{l}\text { Conventionalize } \\
\text { d forms }\end{array}$} & Interrogative Form & 19 & 9 & 15 & 6 & 18 & 9 & 2 & 16 & 24 & 7 \\
\hline & & $\begin{array}{l}\text { Possibility/Probabilit } \\
\text { y }\end{array}$ & 12 & 15 & 10 & 13 & 3 & 8 & 20 & 7 & 3 & 2 \\
\hline & & Should & 1 & 7 & 17 & 16 & 22 & 16 & 11 & 10 & 19 & 18 \\
\hline & & Need & 9 & - & 10 & 1 & 4 & 2 & 2 & 12 & 4 & 3 \\
\hline & & Conditional & 6 & 15 & 14 & 22 & 13 & 9 & 11 & 11 & 11 & 23 \\
\hline & \multirow[t]{4}{*}{ Indirect } & Impersonal & 10 & 20 & 8 & 11 & 5 & 11 & 5 & 12 & 21 & 26 \\
\hline & & Hints & - & - & - & - & - & - & - & - & - & - \\
\hline & & Missing answers* & - & - & - & - & 2 & 8 & 5 & - & - & - \\
\hline & & Total & 96 & 96 & 96 & 96 & 94 & 88 & 91 & 96 & 96 & 96 \\
\hline
\end{tabular}

* Number of the participants who had not written any answers

The findings mentioned in the tables indicate that both $3^{\text {rd }}$ and $4^{\text {th }}$ graders utilized the same suggestion strategies (conventionalized forms).

\subsection{Findings for the fourth research question}

Finally, the responses of the participants for the last research question highlight that the participants mostly preferred conventionalized forms of suggestions. No remarkable distinction between ages was observed and they had similar attitudes and preferences (Table 10-11).

Table 10: Preferences of the participants in terms of their age groups

\begin{tabular}{|c|c|c|c|c|c|c|c|c|c|c|c|c|}
\hline & & Suggestion Type & $\begin{array}{l}\mathrm{Q}_{1} \\
(f)\end{array}$ & $\begin{array}{l}\mathrm{Q}_{2} \\
(f)\end{array}$ & $\begin{array}{l}\mathrm{O}_{3} \\
(f)\end{array}$ & $\begin{array}{l}\mathrm{O}_{4} \\
(f)\end{array}$ & $\begin{array}{l}\mathrm{O}_{5} \\
(f)\end{array}$ & $\begin{array}{l}\text { Q6 } \\
(f)\end{array}$ & $\begin{array}{l}\text { Q7 } \\
(f)\end{array}$ & $\begin{array}{l}\text { Q8 } \\
(f)\end{array}$ & $\begin{array}{l}\text { Q }_{9} \\
(f)\end{array}$ & $\begin{array}{c}0 \\
10 \\
(f)\end{array}$ \\
\hline \multirow{13}{*}{ 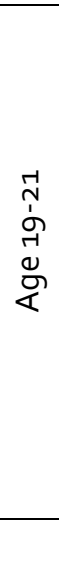 } & \multirow{4}{*}{ Direct } & Performative Verb & 19 & 14 & 17 & 4 & 3 & 2 & 3 & 8 & 9 & 3 \\
\hline & & Noun of Suggestions & 9 & - & - & - & 21 & 7 & 11 & 1 & 1 & 3 \\
\hline & & Imperative & 1 & 22 & 1 & - & - & 15 & 7 & 2 & 1 & 1 \\
\hline & & Negative Imperative & - & - & - & 14 & - & - & 13 & 16 & 5 & - \\
\hline & \multirow{5}{*}{ Conventionalized } & Interrogative Form & 7 & 8 & 14 & 5 & 14 & 12 & 5 & 13 & 17 & 7 \\
\hline & & Possibility/Probability & 9 & 13 & 13 & 19 & 2 & 5 & 13 & 5 & 4 & 1 \\
\hline & & Should & - & 2 & 10 & 14 & 22 & 8 & 6 & 10 & 16 & 10 \\
\hline & & Need & 14 & 3 & 5 & 5 & - & 6 & - & 10 & 1 & 6 \\
\hline & & Conditional & 9 & 5 & 12 & 13 & 6 & 7 & 15 & 6 & 15 & 25 \\
\hline & \multirow{4}{*}{ Indirect } & Impersonal & 10 & 11 & 6 & 4 & 9 & 11 & 4 & 7 & 9 & 21 \\
\hline & & Hints & - & - & - & - & - & - & - & - & - & - \\
\hline & & Missing answers* & & & & & 1 & 5 & 1 & & & 1 \\
\hline & & Total & 78 & 78 & 78 & 78 & 77 & 73 & 77 & 78 & 78 & 77 \\
\hline
\end{tabular}

* Number of the participants who had not written any answers 
Table 11: Preferences of the participants in terms of their age groups

\begin{tabular}{|c|c|c|c|c|c|c|c|c|c|c|c|c|}
\hline & & Suggestion Type & $\begin{array}{l}\mathrm{Q}_{1} \\
(f)\end{array}$ & $\begin{array}{l}\mathrm{Q}_{2} \\
(f)\end{array}$ & $\begin{array}{l}\mathrm{O}_{3} \\
(f)\end{array}$ & $\begin{array}{l}\mathrm{O}_{4} \\
(f)\end{array}$ & $\begin{array}{l}\mathrm{O}_{5} \\
(f)\end{array}$ & $\begin{array}{l}\text { Q6 } \\
(f)\end{array}$ & $\begin{array}{l}\text { Q7 } \\
(f)\end{array}$ & $\begin{array}{l}\text { Q8 } \\
(f)\end{array}$ & $\begin{array}{l}\text { Q9 } \\
(f)\end{array}$ & $\begin{array}{c}\mathrm{Q}_{10} \\
(f)\end{array}$ \\
\hline \multirow{13}{*}{ 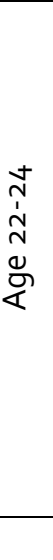 } & \multirow{4}{*}{ Direct } & Performative Verb & 21 & 12 & 17 & 15 & 8 & 6 & 9 & 15 & 8 & 3 \\
\hline & & Noun of Suggestions & 6 & - & - & 1 & 21 & 3 & 8 & 2 & - & 11 \\
\hline & & Imperative & - & 12 & - & - & - & 22 & 7 & - & - & - \\
\hline & & Negative Imperative & - & - & - & 11 & - & - & 14 & 8 & 6 & - \\
\hline & \multirow{5}{*}{ Conventionalized } & Interrogative Form & 17 & 10 & 14 & 5 & 17 & 9 & 2 & 14 & 20 & 6 \\
\hline & & Possibility/Probability & 10 & 10 & 10 & 12 & 1 & 4 & 14 & 4 & 1 & 1 \\
\hline & & Should & 1 & 6 & 12 & 11 & 16 & 11 & 11 & 10 & 15 & 16 \\
\hline & & Need & 9 & 1 & 9 & 2 & 4 & 1 & 2 & 11 & 4 & 4 \\
\hline & & Conditional & 5 & 10 & 10 & 14 & 8 & 7 & 7 & 7 & 8 & 16 \\
\hline & \multirow{4}{*}{ Indirect } & Impersonal & 11 & 19 & 8 & 9 & 4 & 12 & 2 & 9 & 18 & 22 \\
\hline & & Hints & - & - & - & - & - & - & - & - & - & - \\
\hline & & Missing answers* & & & & & 1 & 5 & 4 & & & 1 \\
\hline & & Total & 80 & 80 & 80 & 80 & 79 & 75 & 76 & 80 & 80 & 79 \\
\hline
\end{tabular}

* Number of the participants who had not written any answers

In response to research question 4, nearly all of those participants preferred the use of conventionalized forms of suggestion.

\subsection{Interview}

Concerning the interview findings, the data provided a profound insight. The results can be summarised as follows:

The great majority of the participants seemed to be inclined to offer help to people without discriminating what kind of problem they have. Overall scrutiny of the situations the participants encountered put forward that age, gender, and the level of intimacy with a given interlocutor generally influenced the participants' attitudes, preferences and type of suggestions while making suggestions.

When tabulating the factors affecting participants' attitudes and preferences to make suggestions, the interlocutors' gender has taken the first place. While some female participants (e.g., S3, S8, S10, S12, S16, S17, S18, S21, S22, S24, S25, S26) felt more comfortable with the same gender interlocutors, some male participants (e.g., $\mathrm{S}_{2}, \mathrm{~S}_{5}, \mathrm{~S}_{9}$, $\mathrm{S}_{13}, \mathrm{~S}_{14}, \mathrm{~S}_{15}$ ) decided to be more polite and more selective while making suggestions with female interlocutors. Additionally, it should also be pointed out that only a few participants (e.g., $\left.\mathrm{S}_{2}(\mathrm{~F}), \mathrm{S}_{18}(\mathrm{~F}), \mathrm{S} 6(\mathrm{M}), \mathrm{S}_{15}(\mathrm{M})\right)$ mentioned that they did not take into account the gender of the participants.

Interlocutors' age took the second place for participants when they wanted to suggest something. A majority of the participants (e.g., $\mathrm{S}_{1}(\mathrm{~F}), \mathrm{S}_{2}(\mathrm{M}), \mathrm{S}_{3}(\mathrm{~F}), \mathrm{S}_{7}(\mathrm{~F}), \mathrm{S}_{8}(\mathrm{~F}), \mathrm{S}_{13}(\mathrm{M})$, $\left.\mathrm{S}_{14}(\mathrm{M}), \mathrm{S}_{19}(\mathrm{~F}), \mathrm{S}_{20}(\mathrm{~F})\right)$ stated that they felt at ease with interlocutors who were younger than them. But additionally, a small minority of the participants (e.g., $\mathrm{S}_{4}(\mathrm{~F}), \mathrm{S}_{5}(\mathrm{M}), \mathrm{S} 6$ (F), S26 (F)) elucidated that they were not concerned with the interlocutors' age since they had concentrated on the situation and the problem only.

Finally, the degree of kinship, intimacy or sincerity with an interlocutor, played another major role in the participants' attitude and decision to make a suggestion. Some participants ( e.g. 
$\left.\mathrm{S}_{3}(\mathrm{~F}), \mathrm{S}_{7}(\mathrm{~F}), \mathrm{S}_{9}(\mathrm{M}), \mathrm{S}_{11}(\mathrm{M}), \mathrm{S}_{18}(\mathrm{~F}), \mathrm{S}_{22}(\mathrm{~F}), \mathrm{S}_{25}(\mathrm{~F})\right)$ stated that those factors did not much influence their decision for tackling a problem, but on the other hand, most of the female participants ( e.g. S1 (F), S2 (M), S8 (F), S12 (F), S14 (M), S16 (F), S21 (F), S23 (F), S 24 (F), S 27 $(F))$ believe that peoples' degree of kinship, level of intimacy or sincerity with someone influenced their preferences in adopting an appropriate strategy for suggesting a situation. From those responses, it can be concluded that females were more sensitive and tolerant for elder people rather than males.

\section{Discussion}

Pertaining to the first research question, the results demonstrated that conventionalized forms were the most preferred suggestion types for both males and females because students frequently use them in their daily interactions. Results are noticeably in line with the study of Liu \& Zhao (2007), who considered modals as one of the most frequent strategies by both natives and EFL learners. Importantly, it should also be emphasized here that females additionally employed more "indirect strategies" rather than the males. This can be ensured that the female language is less forceful and indirect (Crawford et al., 1983; Quadoury Abed, 2011), while that of men is assertive and direct (Lakoff, 1973, 1975). This finding has been supported by Al-Kayed \& Al-Ghoweri (2019), who declared that Jordanians had adopted more indirect strategies. Additionally, as stated by Pishghadam and Sharafadini (2011a), Zainall Arif \& Mugableh (2013), Rezvani et al. (2017), Sharqawi \& Anthonoy (2019), there were statistically differences in terms of gender in their use of suggestions.

Regarding the second research question, it was observed that having been graduated from different high schools did not much influence the preference of the participants. While the average response of "direct forms of suggestion" is $56.9 \%$ for Anatolian High School students, it is $22.8 \%$ for Anatolian Imam Hatip High School students and $20.2 \%$ for Anatolian Teacher's High School students. Liu \& Zhao (2007); Farnia et al. (2014) and Pishghadam \& Sharafadini $(2011 b)$ stated that non-native speakers produced more direct strategies in realizing speech acts of suggestion. In this context, to prefer using "direct" strategies, as opted by Yıldız (2020), could be regarded as face-threating by the hearers; therefore, a great majority of the participants had not decided to use direct strategies. In this respect, the use of direct forms of suggestions are not welcomed in Turkish culture, especially for older people, and they may be accepted as less pushy. This view has shown similarities with the findings of Visiaty and Piantari's (2019) study that put forward that the cultural backgrounds of the participants highly affected their choices.

For the third research question, $3^{\text {rd }}$ graders employed "interrogative form, possibility/probability, should and need" respectively, $4^{\text {th }}$ graders preferred the use of conditional strategies. As the least commonly used ones were "negative imperative" for $3^{\text {rd }}$ graders and "imperative" for $4^{\text {th }}$ graders. Additionally, the distribution of "performative verb, nouns of suggestions, imperative, negative imperative, interrogative form, and need" between $3^{\text {rd }}$ and $4^{\text {th }}$ graders were different from each other. It must be emphasized here that the preference of using imperatives in some situations may be the reason of the interlocuters were one of their classmates.

Finally, regarding the last research question, the age of the participants did not create any remarkable results that influenced their preferences. Because their ages were approximately equal, they were expected to have similar or close attitudes, values and preferences. 
Depending upon research conducted by Önalan \& Çakır (2018), the younger generation in Turkey was stated as becoming more direct as a result of the fact that they were more exposed to western cultural values because it was believed that directness was perceived as relevant rather than impoliteness in western culture. But the results of this study are not in line with the findings of that study.

\section{Limitations of the Study}

Firstly, the selection of the participants only from $3^{\text {rd }}$ and $4^{\text {th }}$ graders of the ELT department may not be sufficient enough to deduce remarkable data about the forms of suggestion preferences of Turkish ELT students. Secondly, some situations in the WDCT may not be interesting for some students; therefore, some participants may not have given expected answers. Thirdly, this study was conducted with the participation of only one state university. Besides these factors, the validation of WDCT has not been done by real native speakers.

\section{Conclusion and Suggestions}

To sum up, conventionalized forms of suggestions were frequently used by the participants for every situation without regarding the status of the interlocuters because they were among the most preferable, the most easily remembered words and more frequently used forms of suggestion for non-native speakers of English in Turkey.

Regarding some pedagogical implications, it should be noted here that Turkish learners' similarity or closeness with the interlocuters highly influence their social interaction and communication so that they may neglect or ignore the use of required forms of suggestions (Şanal, 2016). Additionally, the cultural etiquette of the participants influences their perceptions about forms of suggestions. Hence, it appears that Turkish learners are not successful in translating their declarative knowledge into procedural knowledge because of their cultural routine. Lack of or limited knowledge about strategic, discourse and sociolinguistic competencies may have affected the participants' choices to make suggestions. Critical attention should also be paid to the mother tongue interference and negative transfer from L1 to L2. Hence, courses that will enhance their social interaction, communication, oral productivity, authentic language, critical thinking, etc., may also be useful for students to become effective users of the target language.

For further studies, firstly, participants from other language departments, including the private universities, may be invited to the study. It may also be suggested to include participants from some other departments such as engineering, medicine, agriculture, etc. Real native speakers can be asked to prepare situations to make them more realistic, and also, the validation of WDCT can be done by real native speakers, if any. Different from the variables used in this study, the socioeconomic status of the participants and their academic success scores may also be evaluated.

\section{References}

Ahmadian, M.J., \& Vahid Dastjerdi, H. (2010). A Comparative Study of Perception of Politeness of American Reprimands by Iranian EFL Learners and Americans. The Social Sciences, 5(4), 177-185.

Al-Eryani, A.A. (2007). Refusal Strategies by Yemeni EFL Learners. Asian EFL Journal, 9(2). 19-34. 
Al-Kayed, M., \& Al-Ghoweri, H. (2019). A Sociopragmatic Study of Speech Act of Criticism in Jordanian Arabic. European Journal of Scientific Research, 153(1), 105-117.

Allami, H. (2006). A Sociopragmatic Analysis of Griping: The Case of Iranian Students. The Linguistic Journal. 1(1), 59-76.

Allami, H., \& Naeimi, A. (2010). A Cross-linguistic Study of Refusals: An Analysis of Pragmatic Competence Development in Iranian EFL Learners. Journal of Pragmatics, 43(1), 385-406.

Al-Kahtani, S. W. (2005). Refusal Realisations in Three Different Cultures: A Speech Act Theoretically-based Cross-cultural Study. Journal of King Saud University, 18, 35-57.

Alzeebaree, Y. \& Yavuz, M. A. (2017). Realisation of the Speech Acts of Request and Apology by Middle Eastern EFL Learners. EURASIA Journal of Mathematics, Science and Technology Education. 13(11), 7313-7327.

Amelia Maldin, S. (2019). Suggestion Acts Performed by English Instructors of Conversation Class in Language Centre of Andalas University. Lingua Didactika, 12(2), 109-127.

Aminifard, Y., Safaei,E., \& Askari, H. (2014). Speech Act of Suggestion Across Language Proficiency and Gender in Iranian Context. International Journal of Applied Linguistics \& English Literature. 3(5).

Austin, J. (1962). How To Do Things with Words. Oxford University Press.

Basra, S.M., \& Thoyyibah, L. (2017). A Speech Act Analysis of Teacher Talk in EFL Classes. International Journal of Education, 10(1), 73-81.

Behnam, B., Ali Akbari Hamed, L., \& Goharkhani Asli, F. (2013). An Investigation of Giving Condolences in English and Persian via Short Messages. Procedia: Social and Behavior Sciences, 70, $1679-1685$.

Blum-Kulka, S. (1982). Performing Speech Acts in a Second Language. In S. Blum-Kulka, R. Nir, \& Y. Tobin (Eds.), Issues in the Study of Discourse, 144-177. Academon.

Blum-Kulka, S. (1982). Learning How to Mean in a Second Language: A Study of The Speech Act Performance of Learners of Hebrew as a Second Language. Applied Linguistics, 3(1), 3059.

Goody (Ed.), Questions on Politeness: Strategies in Social Interaction. Cambridge University Press.

Brown, P., \& Levinson, S. (1987). Politeness: Some Universals in Language Usage. Cambridge University Press.

Brown, J.D. (2001) Pragmatics Tests: Different Purposes, Different Tests. In K.R. Rose and G. Kasper (Eds.) Pragmatics in Language Teaching. 301-325. Cambridge University Press.

Bryant Smith, J. (2009). The Acquisition of Pragmatic Competence: Complement Response Strategies in Learners of Spanish. A Doctoral Dissertation. Louisiana State University.

Cheng, L. (2009). Indirectness Scale in Chinese Request Speech Act. Us-China Foreign Language, $7(10), 51-60$.

Chiristianto, D. (2020). Speech Acts in EFL Classrooms. Journal Of Pragmatics Research, 2(1), 68-79.

Cohen, A. D. (1996). Speech Acts. In S. L. Mckay \& H. N. Hornberger (Eds.). Sociolinguistics and Language Teaching. 383-420. Cambridge University Press.

Cohen, A.D., \& Olshtain, E. (1993) The Production of Speech Acts by ESL Learners. TESOL Quarterly, 27(1), 33-56.

Crawford, M., Chaffin, R., \& Glenn, J. (1983). Male and Female Language in a PictureDescription Task. Philadelphia, Pa: Paper Presented at the Meeting of the Eastern Psychological Association. 
Eslami-Rasekh, A., \& Mardani, M. (2010). Investigating the Effects of Teaching Apology Speech Act, with a Focus on Intensifying Strategies, on Pragmatic Development of EFL Learners: The Iranian Context. Journal Of Language Society and Culture, 30, 96-103.

Farnia, M., Sohrabie, A. \& Abdul Sattar, H.Q. (2014). A Pragmatic Analysis of Speech Act of Suggestion among Iranian Native Speakers of Farsi. Journal of ELT and Applied Linguistics (JELTALl), 2(2), 48-61.

Gahrouei, V. M. (2013). A Sociopragmatic Study of Speech Act of Suggestion in Persian EFL Learners. Iranian EFL Journal, 9(1).

Görmezler, T. (2016). An Investigation of the Refusal Speech Act of Turkish Learners of English. Unpublished Master Thesis. Kansas State University.

Grundy, P. (2008). Doing Pragmatics ( $3^{\text {rd }}$ ) Edition. Hodder Education.

Gu, T. (2014). A Corpus-Based Study on the Performance of the Suggestion Speech Act by Chinese EFL Learners. International Journal of English Linguistics, 4(1).

Harris, S., Grainger, K., \& Mullany, L. (2006). The Pragmatics of Political Apologies. Discourse \& Society, 17(6), 715-737.

Hudson, T., Detmer, E., \& Brown, J.D. (1995). Developing Prototypic Measures of CrossCultural Pragmatics. University of Hawaii Press.

Jalilifar, A. (2009). Request Strategies: Cross- Sectional Study of Iranian EFL Learners and Australian Native Speakers. English Language Teaching, 2(1), 46-61.

Jiang, X. (2006). Suggestions: What should ESL Students Know? System, 34(1), 36-54.

Karimnia, A., \& Afghari, A. (2010). On The Applicability of Cultural Scripts in Teaching L2 Compliments. English Language Teaching, 3(3), 71-80.

Karagöz-Dilek, T. (2020). Pragmatic Transfer in Turkish EFL Learner's Compliments and Responses from L1 Turkish to L2 English. Journal Of Language and Linguistic Studies, 16(3), 1264-1281.

Kasper, G. \& Dahl, M. (1991) Research Methods in Interlanguage Pragmatics. Studies in Second Language Acquisition, 13(2), 215-247.

Lakoff, R. (1973). The Logic of Politeness, or Minding your P's and Q's. Chicago Linguistic Society, 9, 292-305.

Lakoff, R. (1975). Language and Women's Place. Harper and Row Publishers.

Lenchuk, I. \& Ahmed, A. (2019). Are the Speech Acts of EFL Learners Really Direct? The Case of Request in the Omani EFL Context. SAGE, 1-13.

Li, S. E. (2010). Making Suggestions: A Contrastive Study of Young Hong Kong and Australian Students. Journal of Pragmatics, 42, 598-616.

Liu, Y., \& Zhao, J. (2007). Suggestions in Teacher-Student Conferences. Arizona Working Papers in SLA and Teaching, 14, 59-74.

Martinez-Flor, A. (2004). "The Effect of Instruction on the Development of Pragmatic Competence in the English as a Foreign Language Context: A Study Based on Suggestions." Doctoral Dissertation. Universitat.

Martinez-Flor, A. (2005). A Theoretical Review of The Speech Act of Suggesting: Towards a Taxonomy for its Use in Flt. Revista Alicantina De Estudios Ingleses, 18(1),167-187.

Martinez-Flor, A., \& Uso-Juan, E. (2006). Pragmatic Development in a Second or Foreign Language: Some Classroom Technique. Greta 14(1), 50-56.

Martinez-Flor, A. (2003). Non-Native Speakers' Production of Advice Acts: The Effects of Proficiency." Revista Electronica De Linguistica Applicada, 16 (2), 139-153. 
Martinez-Flor, A. (2006). Task Effects on EFL Learners' Production of Suggestions: A Focus on Elicited Phone Messages and Emails. Journal of English and American Studies, 33, 4764 .

Martinez-Flor,A., \& Alcon Soler, E.(2004). Developing Pragmatic Awareness of Suggestions in the EFLClassroom. Canadian Journal of Applied Linguistics, 10(1), 47-76.

Martinez-Flor, A., \& Fukuya, Y. J. (2005). The Effects of Instruction on Learners' Production of Appropriate and Accurate Suggestions. SYSTEM, 33(3), 463-480.

Matsumura, Sh. (2001). Learning the Rules for Offering Advice: A Quantitative Approach to Second Language Socialization. Language Learning, 51(4), 635-679.

Nguyen, T.M.P. (2007). Cross-Cultural Pragmatics: Refusals of Requests by Australian Native Speakers of English and Vietnamese Learners of English. Published Ma Thesis in Tesol Studies. The University of Queensland.

Nugroho, M., Tarjana, S. S.,\& Purnanto, D. (2018). The Speech Act of Suggestion by Islamic Preachers (Da'i) in Dialogic Da'wah in the City of Surakarta. Advances in Social Science, Education and Humanities Research. 166, 401-405.

Önalan, O. \& Çakır, A. (2018). A Comparative Study on Speech Acts: Formal Complaints by Native Speakers and Turkish Learners of English. Eurasian Journal of Applied Linguistics, 4(2), 239-259.

Parvaresh, V., \& Eslami Rasekh, A. (2009). Speech Act Disagreement among Young Women in Iran. Comparative Literature and Culture, 11(4), 2-8.

Pishghadam, R. \& Sharafadini, M. (2011a). Delving into Speech Act of Suggestion: A Case of Iranian EFL Learners. International Journal of Business and Social Science, 2(16), 152-16o.

Pishghadam, R., \& Sharafadini, M. (2011b). A Contrastive Study into the Realisation of Suggestion Speech Act: Persian vs. English. Canadian Social Science, 7(4), 230-239.

Quadoury Abed, A. (2011). Pragmatic Transfer in Iraqi EFL Learners' Refusals. International Journal of English Linguistics, 1(2), 66-185.

Rezvani, M., Ismael, A. D., \& Tok, S. (2017). Speech Act of Refusal among English Language Students. International Journal of Research in Teacher Education, 8(2), 1-11.

Rose, K.R. \& Ono, R. (1995) Eliciting Speech Act Data in Japanese: The Effect of Questionnaire Type. Language Learning, 45(2), 191-223.

Sabzalipour, B., Mansour K., M., \& Akbar A., A. (2017). Investigation the Effect of Colloconstructural Corpus-based Instruction on Pragmalinquistic Knowledge of Request Speech Act: Evidence from Iranian EFL Students.Special Issue on Language \& Literature, 6(7)

Salam El-Daks, D. A., Ambreen, F., Zaheer, M., \&Gusarova, Y. (2019). A Pragmatic Analysis of the Speech Act of Critcizing in University Teacher-Student Talk. The Case of English as aLingua Franca. Pragmatics, 29(4),493-520.

Schmidt, R. W., \& Richards, J. C. (1980). Speech Acts and Second Language Learning. Applied Linguistics, 1(2), 129-157.

Shahreza, P. (2013). A Sociolinguistic and Cross-Cultural Investigation into the Speech Act of Suggestion. International Journal of Culture and History, 1(1).

Sharifian, F. (2005). The Persian Cultural Schema of "Shekasteh-Nafsi": A Study of Compliment Responses in Persian and Anglo-Australian Speakers. Pragmatics and Cognition, 13(2), 337-362. 
Sharifian, F. (2008). Cultural Schemas in L1 and L2 Compliment Responses: A Study of Persian-Speaking Learners of English. Journal of Politeness Research. Language, Behaviour, Culture, 4(1), 55-80.

Sharqawi, M. A., \& Anthony, E. M. (2020). Analysing Gender Effect on The Speech Act of Suggestion: A Pilot Study. Academic Journal of Interdisciplinary Studies, 9(4).

Sharqawi, M. A. \& Anthony, E. M. (2019). Analysing The Speech Act of Disagreement Produced by Iraqi EFL Learners: A Gender Study. Humanities \& Social Sciences Reviews, $7(2), 118-131$

Shofwan, M. I., \& Mujiyanto, J. (2018). Realisation of Speech Acts of Suggestion by EFL Learners of Universitas Negeri Semarang. English Education Journal, 8(1), 87-95.

Sum-Hung Li, E. (2010). Making Suggestions: A Contrastive Study of Young Hong Kong and Australian Students. Journal of Pragmatics, 42(3), 598-616.

Şanal, M. (2016). Conceptual Socialisation in EFL Contexts: A Case Study on Turkish EFL Learners' Request Speech Acts Realisation. Unpublished Ma Thesis. Bilkent University.

Taguchi, N. (2006). Analysis of Appropriateness in a Speech Act of Request in L2 English. Pragmatics, 16(4), 513-533.

Trosborg, A. (1995). Interlanguage Pragmatics: Request, Complaints, and Apologies. Mouton De Gruyter.

Tuncer, H., \& Turhan, B. (2019). Refusal Strategies of Turkish Pre-Service Teachers of English: A Focus on Gender and Status of the Interlocutor. Journal of Language and Linguistic Studies, 15(1), 01-19.

Tunçel, R. (1999). Speech Act Realisations of Turkish EFL Learners: A Study on Apologizing and Thanking .Unpublished Ph.D. Dissertation. Eskişehir Anadolu Üniversitesi. SBE.

Visiaty, A., \& Piantari, L. L. (2019). Speech Act of Suggestion by Indonesian Students during Peer Response Activities. Proceedings of the 2nd International Conference on Social Sciences And Interdisciplinary Studies, 24-25 Oct., North Sumatera, Indonesia.

Wang, Q. (2019). A Comparative Study of Gender Differences in Refusal Strategies from English Majors. Theory and Practice in Language Studies, 9 (8),1040-1048.

Wannaruk, A. (2008). Pragmatic Transfer in Thai EFL Refusals. RELC, 39(3), 318-337.

Wolfson, N., Marmor, T., \& Jones, S. (1989). Problems in the Comparison of Speech Acts Across Cultures. In S. Blum-Kulka, J. House \& G. Kasper (Eds.), Cross-Cultural Pragmatics: Requests and Apologies, 175-196. Ablex.

Wolfson, N. (1981). Compliments in Cross-Cultural Perspective. TESOL Quarterly, 15(2),117124.

Yelfiza, Y. (2013). Speech Acts for Supporting English Teaching and Learning. International Conference on Language and Arts, Proceeding: International Seminar on Languages and Art, 618-626.

Yıldız, T. E. (2020). A Study on the Use of Suggestion Strategies among Turkish EFL Learners. Tojqi, 11(1), 36-55.

Zainal Ariff, T. N. A., \& Mugableh, A. I. (2013). Speech Act of Promosing among Jordians. International Journal of Humanities and Social Science, 3(13), 248-266. 